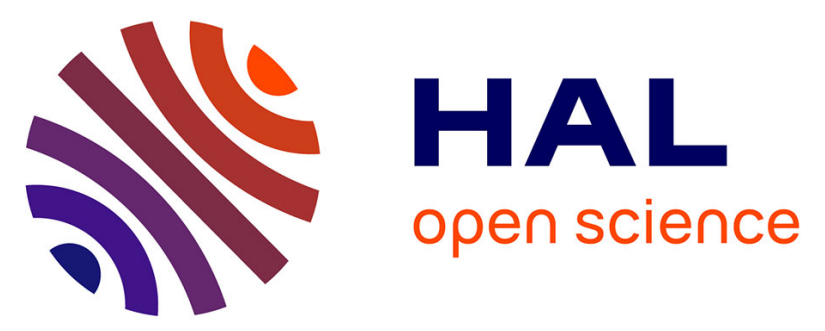

\title{
1H NMR metabonomics can differentiate the early atherogenic effect of dairy products in hyperlipidemic hamsters.
}

Jean-Charles Martin, Cécile Canlet, Bernadette Delplanque, Genevieve Agnani, Denis Lairon, Gaëlle Gottardi, Karima Bencharif, Daniel Gripois, Anissa Thaminy, Alain Paris

\section{To cite this version:}

Jean-Charles Martin, Cécile Canlet, Bernadette Delplanque, Genevieve Agnani, Denis Lairon, et al.. $1 \mathrm{H}$ NMR metabonomics can differentiate the early atherogenic effect of dairy products in hyperlipidemic hamsters.. Atherosclerosis, 2009, 206 (1), pp.127-33. 10.1016/j.atherosclerosis.2009.01.040 . inserm-00370368

\section{HAL Id: inserm-00370368 https://www.hal.inserm.fr/inserm-00370368}

Submitted on 24 Mar 2009

HAL is a multi-disciplinary open access archive for the deposit and dissemination of scientific research documents, whether they are published or not. The documents may come from teaching and research institutions in France or abroad, or from public or private research centers.
L'archive ouverte pluridisciplinaire HAL, est destinée au dépôt et à la diffusion de documents scientifiques de niveau recherche, publiés ou non, émanant des établissements d'enseignement et de recherche français ou étrangers, des laboratoires publics ou privés. 


\section{Accepted Manuscript}

Title: ${ }^{1} \mathrm{H}-\mathrm{NMR}$ metabonomics can differentiate the early atherogenic effect of dairy products in hyperlipidemic hamsters

Authors: Jean-Charles Martin, Cécile Canlet, Bernadette Delplanque, Genevieve Agnani, Denis Lairon, Gaëlle

Gottardi, Karima Bencharif, Daniel Gripois, Anissa Thaminy, Alain Paris

PII: S0021-9150(09)00084-7

DOI: doi:10.1016/j.atherosclerosis.2009.01.040

Reference: ATH 10778

To appear in: Atherosclerosis

Received date: 22-4-2008

Revised date: 29-12-2008

Accepted date: 27-1-2009

Please cite this article as: Martin J-C, Canlet C, Delplanque B, Agnani G, Lairon D, Gottardi G, Bencharif K, Gripois D, Thaminy A, Paris A, ${ }^{1} \mathrm{H}-\mathrm{NMR}$ metabonomics can differentiate the early atherogenic effect of dairy products in hyperlipidemic hamsters, Atherosclerosis (2008), doi:10.1016/j.atherosclerosis.2009.01.040

This is a PDF file of an unedited manuscript that has been accepted for publication. As a service to our customers we are providing this early version of the manuscript. The manuscript will undergo copyediting, typesetting, and review of the resulting proof before it is published in its final form. Please note that during the production process errors may be discovered which could affect the content, and all legal disclaimers that apply to the journal pertain. 


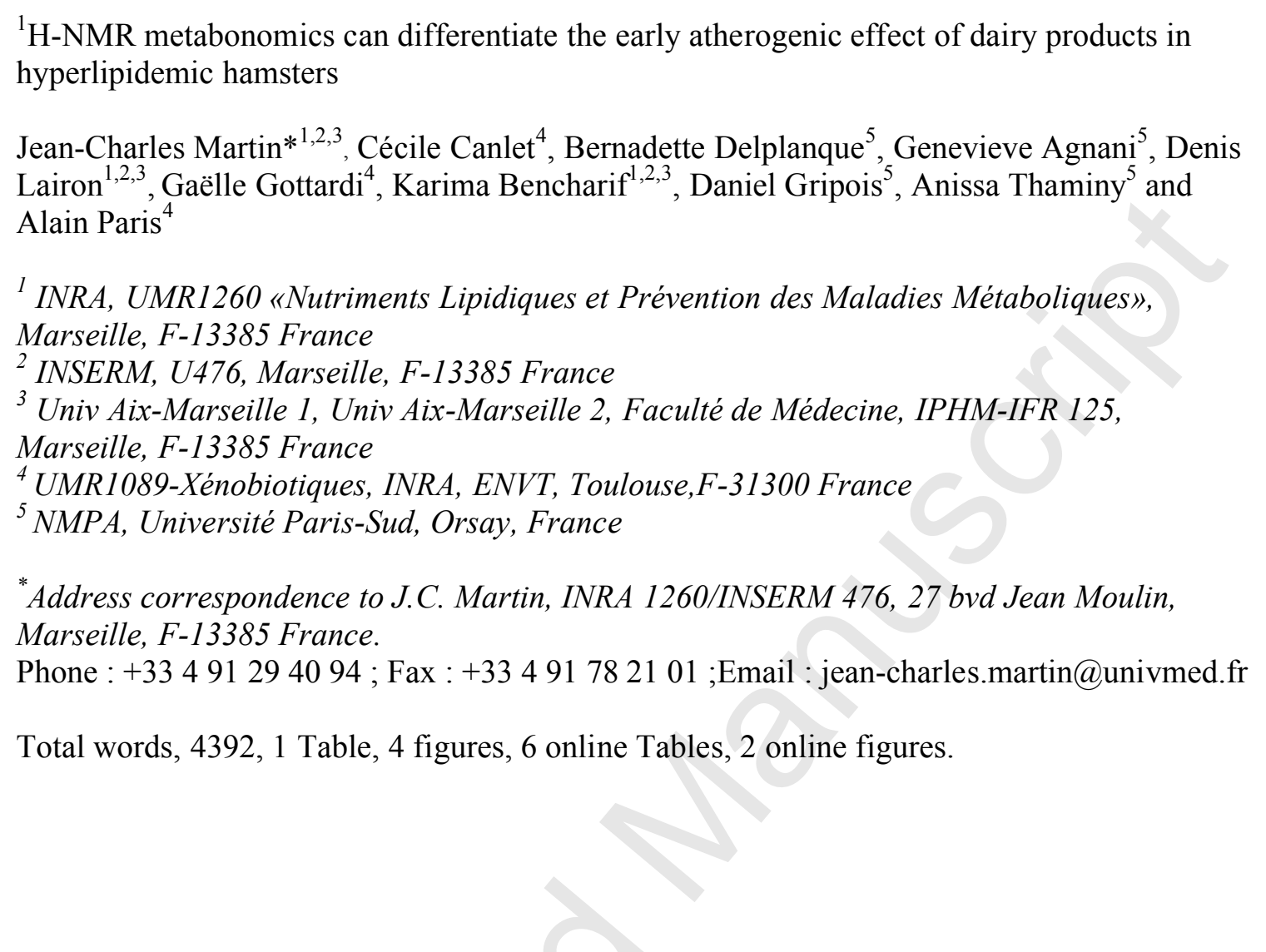




\begin{abstract}
Diet is an important environmental factor modulating the onset of atherosclerosis. The aim of this study was to evaluate the effects of different dairy-based food products on early atherogenesis using both conventional and metabonomic approaches in hyperlipidemic hamsters. The hamsters received up to $200 \mathrm{~g} / \mathrm{kg}$ of fat as anhydrous butter or cheese made from various milk fats or canola-based oil (CV), in addition to a non-atherogenic low-fat diet. Aortic cholesteryl ester loading was considered to be an early atherogenic point, and metabolic changes linked to atherogenesis were measured using plasma ${ }^{1} \mathrm{H}-\mathrm{NMR}$-based metabonomics. The lowest atherogenicity was obtained with the plant-oil cheese diet, followed by the dairy fat cheese diet, while the greatest atherogenicity was observed with the butter diet $(P<0.05)$. Disease outcome was correlated with conventional plasma biomarkers (total cholesterol, triglycerides, LDL cholesterol, $R^{2}=0.42-0.60$ ). NMR plasma metabonomics selectively captured part of the diet-induced metabotypes correlated with aortic cholesteryl esters $\left(R^{2}=0.63\right)$. In these metabotypes, VLDL lipids, cholesterol, and $\mathrm{N}$-acetylglycoproteins ( $R^{2}$ range: $\left.0.45-0.51\right)$ were the most positively correlated metabolites, whereas a multimetabolite response at $3.75 \mathrm{ppm}$, albumin lysyl residues, and trimethylamine- $N$-oxide were the most negatively correlated metabolites $\left(R^{2}\right.$ range: $\left.0.43-0.63\right)$ of the aortic cholesteryl esters. Collectively, these metabolites predicted $89 \%$ of atherogenic variability compared to the $60 \%$ predicted by total plasma cholesterol alone. In conclusion, we show that the food environment can modulate the atherogenic effect of dairy fat. This proof-of-principle study demonstrates the first use of plasma metabonomics for improving the prognosis of dietinduced atherogenesis, revealing novel potential disease biomarkers.

Keywords: nuclear magnetic resonance spectroscopy, milk fat, atherosclerosis biomarkers, hamster, metabonomics
\end{abstract}




\section{Introduction}

Dietary fats, particularly saturated fatty acids, have been implicated in the etiology of cardiovascular disease ${ }^{1}$. Current dietary guidelines advise limiting the consumption of saturated fatty acids to no more than $10 \%$ of the total daily energy intake ${ }^{2}$. As milk fat represents a rich source of saturated fatty acids, dairy products are recommended to be consumed sparingly ${ }^{3}$, although this advice has been challenged by recent studies ${ }^{4,5}$. These conflicting results may arise from (i) the high variation of fatty acid composition in milk fat related to atherogenic risk ${ }^{6,7}$, (ii) the type of dairy products consumed (fermented or not) ${ }^{4}$, and (iii) the complexity of the dairy food matrix. In fact, many foods are complex mixtures of molecules that simultaneously induce chronic or subtle modifications in numerous biochemical pathways. Thus, it seems relevant to consider how the dietary environment can modulate the various effects of dairy fats on atherosclerosis. As analysis with a limited number of biomarkers can lead to inaccurate diagnosis of a multifactorial disease, such as atherosclerosis, but a new approach based on metabonomics could provide a suitable tool for examining phenotypes using hundreds of metabolite descriptors 8,9 . Although it is a recent introduction to the field of nutrition research, nutritional metabonomics has already delivered interesting insights into the understanding of metabolic responses of humans or animals in response to dietary interventions, as well as for the definition of metabolic phenotypes ${ }^{10-14}$. Nevertheless, it has not yet been applied to the domain of diet-induced atherosclerosis as a descriptive, nontargeted approach for evaluating the effects of dietary treatment on metabolic phenotypes, which are also called metabotypes ${ }^{15}$. These metabotypes can be related to health outcomes and provide predictive disease or risk assessment biomarkers ${ }^{16},{ }^{17}$, although strong clinical validations are required ${ }^{17,18}$.

Thus, we addressed whether different types of dairy products could affect the outcome of atherogenesis, using both conventional blood chemistry and a metabolome-wide approach. The latter was then used as a proof-of-principle study to measure diet-induced metabolic disruptive events for finding new potential biomarkers of the disease. For this, we used a hamster model that has been previously established for studying diet-induced atherogenesis ${ }^{7}$, 19 .

\section{Materials and Methods}

\subsection{Animals and diets}

All experiments were approved by the referee for animal care at our institution and conducted in accordance with the French Regulations for Animal Experimentation (Art 19.Oct 1987, Ministry of Agriculture). Forty-eight male Golden Syrian hamsters (5-week-old Janvier strain weighing $67 \pm 1 \mathrm{~g}$ ) were purchased from the Janvier Breeding Center (Le Genest-St Isle, France). Each hamster was individually caged and fed a commerciallyavailable chow diet (25/18 standard diet from Mucedola, Settimo, Milanese, Italy) for a oneweek adaptation period. Eight hamsters per group were then fed experimental diets, gradually increasing the fat content from a $100 \mathrm{~g} / \mathrm{kg}$ diet for five weeks to a $200 \mathrm{~g} / \mathrm{kg}$ diet for the next twelve weeks. Control hamsters were continuously fed the chow-based diet. The various fats were delivered as lyophilized cheeses made of canola-based oil (CV), pasteurized milk fat (CS), olein milk fat fraction (CO), or raw milk fat (CR). A fifth group was given fat as anhydrous butter from milk used in the making of CS. The low atherogenic reference diet was the low-fat, basal chow diet ${ }^{19}$. Diet and water were available ad libitum, and the cheeses were provided by Lactalis (Retiers, France). The fatty acid compositions of the various products and of the basal diet are listed in Tables 1, 2 and 3 of the Online Supporting Material. 
2.2. Blood sampling and chemical assays

Hamsters were anesthetized 5-8 $\mathrm{h}$ after their last meal by an intramuscular injection of Zoletil $^{\circledR} 50$ at a dose of $250 \mathrm{mg}$ per kg. This corresponded to a late post-prandial situation. A cardiac puncture was used to collect 3-4 $\mathrm{mL}$ of blood, and plasma aliquots were stored at $80^{\circ} \mathrm{C}$ until ready for analysis.

Plasma lipids were measured by enzymatic procedures using the following commercial kits (Biomerieux, Lyon, France): total cholesterol (RTU method) and triglycerides and phospholipids (PAP 150 method) ${ }^{7}$.

Lipoproteins were fractionated from 0.4 mLof the plasma samples by density gradient ultracentrifugation ${ }^{19}$.

Whole aortic lipids were extracted and analyzed by gas liquid chromatography using a high temperature non-polar capillary column ${ }^{7,19}$. Whole aortic cholesteryl esters were taken as an indicator of early atherogenesis (fatty streaks) ${ }^{19,20}$, and expressed as a \% of the tissue phospholipids. The method was adapted from Eggen et al. ${ }^{21}$ and Kuksis et al. ${ }^{22}$. Aortas were excised after rinsing with $5 \mathrm{~mL}$ of saline perfused from the left ventricle, blotted, weighed and stored immediately at $-80^{\circ} \mathrm{C}$ before processing. Aorta tissue was ground with a turrax homogenizer at the maximum speed, first for 1 min with $1 \mathrm{~mL}$ of methanol and $30 \mu \mathrm{g}$ of trimyristine as an internal standard, second with $2 \mathrm{~mL}$ of added chloroform. The probe was rinsed with $0.5 \mathrm{~mL}$ of chloroform:methanol $(2: 1, \mathrm{v} / \mathrm{v})$, and the pooled phase was centrifuged at $1500 \mathrm{rpm}$ for $5 \mathrm{~min}$. The supernatant was removed and filtered on glass wool, and the pellet re-extracted with $1 \mathrm{~mL}$ of chloroform:methanol $(2: 1, \mathrm{v} / \mathrm{v})$. The pooled supernatant was then dried under a stream of nitrogen. The residue was solubilized with $1.2 \mathrm{ml}$ diethyl ether, and 1 $\mathrm{ml}$ Tris $\mathrm{HCl}$ buffer (17.5 mM, pH 7.3) was then added, followed by $2 \mathrm{ml}$ of a solution $(1 \mathrm{U} / \mathrm{ml}$ in Tris buffer, $\mathrm{pH}$ 7.3) of phospholipase C (type III, Sigma). The mixture was incubated at $32^{\circ} \mathrm{C}$ for $2 \mathrm{~h}$ with shaking. The lower organic phase was collected and dried under a vacuum $\left(40^{\circ} \mathrm{C}\right)$. The dry residue was redisolved in $300 \mathrm{ml}$ of hexane, and $1 \mu 1$ was analyzed underivatized by GLC on a short nonpolar capillary column ( $8 \mathrm{~m}$ length, $0.32 \mathrm{~mm}$ inner diameter, $0.25 \mu \mathrm{m}$ film thickness, HP5, Hewlett- Packard; Les Ulis, France). Appropriate response factors were calculated using pure standards.

\subsection{Metabolomic analysis}

Plasma ${ }^{1} \mathrm{H}$ NMR spectroscopy data acquisition was followed by raw data processing (binning), multivariate statistics, NMR variable selection and assignment of metabolite structures (biomarkers identification), and biological interpretation.

\subsection{1. ${ }^{1} H$ NMR spectroscopy}

${ }^{1} \mathrm{H}$ NMR spectra were acquired on a Bruker Avance DRX 600 spectrometer operating at $600.13 \mathrm{MHz}$ at $300 \mathrm{~K}$ and equipped with a triple axis inverse (TXI) gradient cryoprobe. Plasma samples $(500 \mu \mathrm{l})$ were diluted with $\mathrm{D}_{2} \mathrm{O}(100 \mu \mathrm{l})$ to provide a field-frequency lock. ${ }^{1} \mathrm{H}$ NMR spectra were acquired using the Carr-Purcell-Meiboom-Gill (CPMG) spin-echo sequence $\left[{ }^{23}\right.$ with presaturation. These spectra were measured using a spin-echo loop time $(2 \mathrm{n} \tau)$ of $43.9 \mathrm{~ms}$ and a relaxation delay of $2.5 \mathrm{~s}$. A total of 128 transients were collected into $32 \mathrm{k}$ data points using a spectral width of $12 \mathrm{ppm}$ and an acquisition time of $2.28 \mathrm{~s}$. Prior to Fourier transformation, an exponential line broadening function of $0.3 \mathrm{~Hz}$ was applied to the FID.

The acquired NMR spectra were manually phase- and baseline-corrected and referenced to the chemical shift of the methyl resonance of lactate at $\delta 1.33$. ${ }^{1} \mathrm{H}-{ }^{1} \mathrm{H}$ correlation spectroscopy (COSY) ${ }^{24}$ was performed on selected samples to confirm the ${ }^{1} \mathrm{H}$ assignments. A total of 32 transients were acquired into 4096 data points. A total of 256 increments were measured in F1 using a spectral width of $10 \mathrm{ppm}$ and an acquisition time of $0.28 \mathrm{~s}$ was used. The data were weighted using a sine-bell function in $t_{1}$ and $t_{2}$ prior to FT. 
1 1H-13C heteronuclear single quantum coherence (HSQC) ${ }^{25}$ spectra were collected for 2 selected samples with $1 \mathrm{H}$ detection. A relaxation delay of $2.5 \mathrm{~s}$ was used between pulses, and 3 a refocusing delay equal to $1 / 4^{1} \mathrm{~J}_{\mathrm{C}-\mathrm{H}}(1.78 \mathrm{~ms})$ was employed. A total of 2048 data points with 464 scans per increment and 512 experiments were acquired with spectral widths of $10 \mathrm{ppm}$ in $5 \quad \mathrm{~F} 2$ and $180 \mathrm{ppm}$ in F1. The data were multiplied by a shifted Qsine-bell function prior to FT. 6 2.3.2. Statistics software (Umetrics, Umeå, Sweden). The specific effect of each diet on the plasma metabolome was examined by a Projection on Latent Structure-Discriminant Analysis (PLS$D A$ ). The relationship between metabolite profiles and atherogenic outcome, as well as the identification of early atherogenesis biomarkers, were analyzed by the Partial Least Square (PLS) regression method. Unit of variance scaling or pareto scaling of the data did not substantially modify the analyses. Thus, imported data were mean-centered and scaled in unit of variance (1/SD) for all PLS analyses. Since the primary aim of our study was to capture metabolic variation sensitive to dietary treatments and specifically associated with atherogenic development, data were pre-processed using Orthogonal Signal Correction (OSC) with diet as a correction factor. Simply, this procedure allows adjustment of the calculation to any co-variables that could overshadow the treatment effects ${ }^{26}$. Thus, we were able to remove $42.3 \%$ of the variation that was unassociated with the treatments. Validation of the statistics, together with the robustness of the analyses, are provided (please see Online Supporting Material). Unsupervised hierarchical clustering was performed with Permutmatrix ${ }^{27}$ (www.lirmm.fr/ caraux/PermutMatrix/) using the OSC transformed dataset. Briefly, the effect of each dietary treatment was first examined by a discriminant analysis (PLS-DA) to determine the specific metabotypes. Then, the relationship among the metabotypes and the atherogenic outcomes was calculated by PLS. From this analysis, the most correlated NMR variables to aortic cholesteryl-ester were selected and assigned to the unsupervised hierarchical analysis to examine the metabolites clusters. Then, the NMR variables were assigned to plasma metabolites and biomarker identification was performed. Whenever necessary, a $t$-test and a simple linear or multiple regressions analysis was performed using SAS and StatView, respectively (SAS Institute Inc, Cary, NC, USA). The $\alpha-$ risk of 0.05 was the significance statistical threshold used for these tests. While the PLS-based analysis was performed, statistically relevant results and uncertainty estimates were also calculated by the Jack-knife method (a re-sampling method) with $99 \%$ confidence intervals ${ }^{28}$. 


\section{Results}

3.1. Biochemical features

The various diets had not affected weight gain in the animals at the completion of the experimental period (Table 1). However, the cholesteryl ester (CE) loading of the aorta was clearly influenced by the type of fat incorporated into the diet: the plant fat cheese-based diet (CV high-fat diet) produced less lipid deposition than the low-fat reference diet, followed by the dairy fat cheese-based diets (CR, CO, and the CS high-fat diets), and finally by the anhydrous butter diet (Figure 1). The aorta lipid deposition was positively associated with the plasma total cholesterol $\left(\mathrm{R}^{2}=0.60, P<0.001\right)$, triacylglycerol $\left(\mathrm{R}^{2}=0.51, P<0.001\right)$, LDL-C $\left(\mathrm{R}^{2}=0.55, P<0.001\right)$, and nonHDL/HDL-cholesterol ratio $\left(\mathrm{R}^{2}=0.42, P<0.001\right)$ (Table 1$)$. A multiple regression calculation with these plasma values to whole aortic $\mathrm{CE}$ deposition did not improve the prediction index compared to plasma cholesterol alone, which was the best predictor of atherogenicity in our study.

3.2. Dietary treatments induced specific metabotypes

The NMR spectral range that was examined varied from 0.8 to $8.6 \mathrm{ppm}$ (Figure 1 and Table 4 in the Online Supporting Material). Among the 469 ubiquitous NMR signals, 94.6\% were significantly affected across all dietary treatments $(\mathrm{P}<0.05$, not shown). The discriminant analysis indicated that the different dietary intakes induced specific metabolic patterns (metabotypes) that enabled class assignment for most hamsters (PLS-DA, Figure 2). In addition, the distance among the groups in the two-dimensional plot allowed for the evaluation of dietary challenges on changes in the plasma metabolome. For instance, compared with the low-fat reference diet, the high plant fat cheese diet (CV) induced the least metabolic change, while the anhydrous butter diet induced the greatest change $131.3 \%$, and $66.2 \%$ to control, respectively; $\mathrm{P}<0.05$, not shown). Also, the metabolic signature of the hamsters that were fed raw milk cheese (CR) could be distinguished in the second component t[2] of the PLS-DA plot from the hamsters that had received the same product made from pasteurized milk (CS) (Figure 2).

\subsection{Metabolite profile is correlated with atherogenic outcome}

We examined whether specific patterns of plasma metabolites associated with dietary treatments could be related to the severity of atherogenic outcome, as measured by aortic cholesteryl ester deposition. A PLS regression between the NMR variables and the cholesteryl esters in the aorta revealed that $62.3 \%$ of the variations in cholesteryl ester loading in the aorta can be predicted by different dietary conditions (Figure 3A). By comparison, the top 20 NMR variables most sensitive to dietary treatments differed from the top 20 NMR variables highly correlated to whole aortic cholesteryl esters (Table 5, Online Supporting Material). The statistical analysis validated by jackknifing with a 99\% CI indicated that $13.8 \%(\mathrm{n}=64)$ of the NMR signals were positively associated with cholesteryl ester deposition in the hamster aorta, and $32.8 \%(n=142)$ were negatively associated. Thus, 53\% of variables remained unrelated to aortic cholesteryl esters $(C E)(\mathrm{n}=261)$ (not shown). We selected NMR signals that were positively ( $\mathrm{n}=7, R^{2}$ range $\left.=0.45-0.51\right)$ or negatively $\left(\mathrm{n}=14, R^{2}\right.$ range $=0.63$ to $0.39)$ related to aortic cholesteryl ester deposition as an indicator of atherogenic lesions (Online Supporting Material Table 6). These signals corresponded to the athero- 'positive' and 'negative'-biomarkers, respectively. When combined in a multiple regression model, up to $89 \%$ of the aortic cholesteryl ester deposition can be predicted by variations in these biomarkers. The relative importance of the metabolites to predict the severity of atherogenic outcomes can be ranked using the variable importance in the projection (VIP) of the PLS procedure (Figure 3B), and their relationships were visualized by hierarchical clustering (Figure 3B).

A resonance value corresponding at $\delta=3.75$ ppm appeared negatively associated to $C E$ deposition and was the most important variable in predicting atherogenesis. This 
chemical shift did not correspond to a single compound, but rather to alanine, glucose, glutamine and three unassigned compounds, as indicated by spiking experiments and with an 1H-13C heteronuclear single quantum coherence NMR analysis (see online supporting material). The multiple nature of that composite signal is likely to explain the absence of any significant PLS correlation with other glucose NMR signals ( $\delta=3.33,3.45,3.64,3.78,3.80$, $3.81, \mathrm{ppm})$, or chemically assessed plasma glucose, as well as with other alanine signals at $\delta=1.48 \mathrm{ppm}$ (not shown).

Other than this composite signal, the atheronegative biomarkers were identified to correspond to glutamine and proline, in addition to glycerol, creatine, citrate, albumin lysyl and polyunsaturated fatty acids (Figure 3B). Conversely, the atheropositive biomarkers corresponded to lipids and $N$-acetyl-glycoproteins.

Compared with pasteurized cheese (CS diet), raw milk cheese (CR diet) more systematically decreased the above atheropositive biomarkers, without affecting the atheronegative ones (Figure 3B, and Online Supplemental Table 6). In addition, the decrease in the atheronegative biomarkers in anhydrous butter fed hamsters supports that pasteurized milk fat given as an anhydrous butter diet appeared to be more atherogenic than given as a cheese diet (CS) (Figure 1, Figure 3B, and Online Supplemental Table 6).

\section{Discussion}

This is the first study examining the suitability of plasma metabonomics to determine the severity of diet-induced atherosclerosis. The hyperlipidemic hamster model used in our study has been previously found to be appropriate for studying atherogenesis associated with dietary challenges ${ }^{7,19}$. A group of hamsters fed a low-fat diet was used as a non-atherogenic reference to better emphasize the metabolic disruptions linked to high-fat diets and the onset of the disease.

In the present metabonomic analysis, hamsters were distinguished based on their dietary treatments, which each induced specific metabotypes. The metabotypes could not be related to weight gain differences, since the weight gains were identical among all the hamster groups. Also, the metabolic shifts did not result from the direct assessment of exogenous dietary metabolites, as the metabolites measured were ubiquitous among all the hamsters. Rather, the changes likely arose from endogenous metabolic adjustments elicited by the dietary challenges. This is illustrated by comparing the low-fat reference diet with either the high-fat plant cheese or the high-fat dairy cheese diets. It appears that the fat quality rather than the fat amount appears to have a noticeably greater impact on the metabolic balance. These observations also strongly indicate that different dairy products do not elicit the same biological responses, even though conventional biomarker properties may appear similar. In fact, our analysis took into account hundreds of descriptors, giving a more robust reflection of the impact of dietary treatments on metabolic state than any conventional measurement analysis.

We then examined which part of the metabolic response to dietary challenges could be associated with the severity of the atherogenic outcome. Whole aortic cholesteryl ester was taken as the endpoint of early atherogenesis ${ }^{19,20,29}$. With regard to the atherogenic outcome, the low-fat (reference diet) and high-fat plant-based products $(\mathrm{CV})$ produced less aortic $\mathrm{CE}$ deposition than the high-fat dairy based products. Among the dairy fat-based products, the anhydrous butter diet exhibited the highest atherogenic response. Atherogenic outcome was positively associated with several conventional plasma biomarkers of atherogenic risk, such as total plasma cholesterol, triglycerides, or LDL cholesterol ${ }^{19,30}$. These results agree with other observations ${ }^{7,31}$ and validate our model of diet-induced atherogenesis. Not surprisingly, these plasma lipids also corresponded to the NMR signals found positively associated with the severity of atherosclerosis, which substantiates our metabonomic approach to find early 
1 atherogenic biomarkers. In addition, the metabonomic approach improved early atherogenic 2 lesion predictions by one-third when compared to the use of conventional biomarkers $\left(R^{2}=\right.$

30.89 compared with 0.60 for plasma cholesterol). This improved accuracy could be valuable 4 to optimize preventive and therapeutic modalities. Importantly, the top 20 NMR variables 5 most sensitive to dietary treatments (extracted from the PLS-DA score plot, Figure 2) differed 6 from the top 20 NMR variables mostly related to whole aortic cholesteryl esters (PLS results, 7 Online Supplemental Table 5). This indicates that the variables related to whole aortic 8 cholesteryl esters are not simply surrogate markers of dietary intake, but rather reflect 9 metabolic disruptions related to the onset of atherogenesis. (neoglucogenic amino-acids, such as alanine and glutamine, glucose and unassigned signals) that was mainly associated with glucose metabolism, and was the most negatively associated to atherogenesis $\left(R^{2}=0.63\right)$. This shift was an even better predictor of atherosclerosis in absolute value than lipoprotein lipids, as indicated by the statistical score ranking (VIP). Such a signal, which corresponds to a $\alpha-\mathrm{CH}$, has been already described in the literature ${ }^{32}$ and has also been found to be unassigned using the same NMR method. From our spiking data, it is tempting to associate this signal to alanine. In our hierarchical cluster analysis, this signal also clustered with glutamine, another neoglucogenic amino-acid, and further to creatine, an energy associated metabolite, which together with alanine have been associated to lower atherogenesis in a apoE-/- mouse model ${ }^{33}$. However, our methodological approach obviously does not allow us to confidently conclude which specific metabolite in the $3.75 \mathrm{ppm}$ signal is the most relevant in the atherogenic prediction. Perhaps, a more advanced NMR analysis or a more sensitive technique (LC-MS) would yield better insight.

In the postprandial conditions of our study, plasma glycerol changes are likely due to the lipase release of glycerol from triglyceride-rich particles. This is indicated by the reciprocal changes in the concentrations among glycerol, plasma lipids and VLDL ( $R^{2}$ range from 0.51 to 0.65 according to the multiple NMR chemical shifts, not shown). High plasma glycerol was associated with lower atherogenesis, which could emphasize the importance of the postprandial blood lipid clearance in the etiology of atherogenesis ${ }^{34}$. We identified resonances that corresponded to $\mathrm{N}$-acetyl-glycoproteins, clustering with plasma lipids and VLDL. These compounds have been identified in arthritis, and as late markers of inflammation and the acute phase protein response, ${ }^{35}$ such as apoSAA and CRP ${ }^{36}$. These compounds are also associated with the development of atherogenesis in the current study. Thus N-acetyl-glycoproteins likely reflect an atherogenesis-induced inflammation in our study. Conversely, the lysine groups in albumin were found to be negatively associated with our atherogenic endpoint. Lysine groups in albumin - and apoB100-containing particles, can form advanced glycation end products or be modified by oxidation ${ }^{37,38}$. A low level of free lysyl groups can thus reflect a proatherogenic oxidative stress. This is also supported by a positive relationship between paraoxonase- 1 activity (an anti-oxidative stress enzyme in plasma) and free albumin lysyl residues $\left(R^{2}=0.41\right.$, regression with albumin lysyl chemical shift at $\delta=2.95$, see Online Supplemental Figure 2).

Interestingly, trimethylamine- $N$-oxide (TMAO), which is often derived from di- and trimethylamine during choline ${ }^{39}$ or L-carnitine ${ }^{40}$ metabolism by gut microbiota, was found to be negatively associated with atherogenesis $\left(R^{2}=0.43\right)$. The dairy cheeses and the plant cheese differed essentially in the fat quality, but also displayed variations in plasma TMAO. Thus, differences in TMAO plasma concentration are not likely to result from the differences in dietary intake of choline or L-carnitine in our conditions, since choline and L-carnitine were originally identical among these products. Nevertheless, the dietary modification of the gut microbiota metabolic activity in the pathogenesis of atherosclerosis cannot be confidently inferred from our results and remains to be confirmed. 
1 Thus, our approach can reveal alterations in inflammation, oxidative stress, and plasma lipid

2 metabolism with a single analysis, thereby emphasizing the multifaceted process of

3 atherosclerosis. In addition, the possible implication of the gut microbiota in early

4 atherogenesis cannot be ruled out.

5 Finally, using the variable importance in projection (VIP) calculation of the PLS regression,

6 we determined which group of metabolites provided the best prediction of atherogenic

7 severity. If a causal link between identified risk factors and disease development could be

8 verified, this could help in establishing the main cause of the disease. Therapeutic

9 intervention could thus be drastically improved through specific targeting.

10 Clustering analysis provided a comparison of the relative influence of each diet on the metabotypes linked to the severity of atherogenesis. The greater atheroprotective effect of the high-fat plant cheese diet (CV) was accompanied by an increase in the atheronegative biomarkers $(P<0.05$, online Supplemental figure 3B and Table 6). A likely reason for this lower atherogenic outcome is that the $\mathrm{CV}$ diet provides a higher polyunsaturated fatty acid and phytosterol intake, together with a lower saturated fatty acid and cholesterol intake (compared to the high milk fat diet groups, Online Supplemental Table 2), resulting in an atheroprotective effect ${ }^{41,42}$.

Excluding milk processing, the raw milk cheese diet (CR) and the pasteurized milk cheese diet were identical. Comparatively, the pasteurized milk cheese diet (CS) displayed an increase in the several atheropositive biomarkers $(P<0.05$, Online Supplemental Table 6). Nevertheless, this increase did not account for the differences in atherogenesis between the CS and CR groups. Fatty streaks were the standard atherogenic outcome in this investigation. Perhaps another model with more advanced lesions would reveal larger differences in atherogenicity among raw milk or pasteurized milk products.

It also appears that variations in macro- and micronutrient intake can drastically influence the atherogenic feature of a given dairy fat moiety, as shown between the cheese (CS) and anhydrous butter diets. This would indicate that the detrimental effect of a high saturated fat intake on atherogenesis can be modulated by accompanying compounds or the physicochemical nature of the food matrix, as corroborated by findings that showed a more deleterious effect of butter than cheese on atherogenic risk ${ }^{4}$. The main metabolic differences observed in plasma between these two dairy fat-based diets in our study was a greater decrease in several of the atheroprotective biomarkers following the anhydrous butter diet $(P$ $<0.05$, Online Supplemental Table 6).

In conclusion, we differentiated the biological responses, particularly of multiple metabolic responses related to diet-induced atherogenesis, using an observational metabonomic approach. In addition to the well recognized risk factors (plasma lipids), we identified new predictors of atherogenesis with either positive or negative association with disease progression. It would be interesting to evaluate whether this approach can reveal early biomarkers in a prevention study. Finally, the atheropositive or atheronegative biomarkers identified are most likely valid only for the specific dietary perturbations studied in our animal model. It remains to be shown whether such markers are also predictive for atherosclerosis resulting from genetic and environmental factors. This proof-of-principle study for the use of metabonomics in diet-induced atherosclerosis prediction demonstrated that metabolic profiling of plasma could be more accurate than using common clinical biomarkers to predict atherogenic risk, helping to determine the relative importance of the underlying causes. An improvement in the accuracy of prediction could be valuable in optimizing preventive and therapeutic modalities. This kind of global approach could help health care professionals who are often confronted with selecting either a single risk factor for treatment or treating several risk factors simultaneously and determining their priority. Due to 
1 the ease of sample collection, the applicability of this approach in humans is promising for 2 examining disease progression. 


\section{References}

1 Keys, A., Menotti, A., Karvonen, M. J., Aravanis, C., Blackburn, H., Buzina, R., Djordjevic, B. S., Dontas, A. S., Fidanza, F., Keys, M. H. and et al., The diet and 15-year death rate in the seven countries study, Am J Epidemiol, 1986, 124: 903-915.

2 De Backer, G., Ambrosioni, E., Borch-Johnsen, K., Brotons, C., Cifkova, R., Dallongeville, J., Ebrahim, S., Faergeman, O., Graham, I. and Mancia, G., European guidelines on cardiovascular disease prevention in clinical practice. Atherosclerosis, 2004, 173: 379-389.

3 Schaefer, E. J., Lipoproteins, nutrition, and heart disease, Am. J. Clin. Nutr., 2002, 75: 191-212.

4 Tholstrup, T., Dairy products and cardiovascular disease, Curr Opin Lipidol, 2006, 17: 1-10.

5 Elwood, P. C., Pickering, J. E., Hughes, J., Fehily, A. M. and Ness, A. R., Milk drinking, ischaemic heart disease and ischaemic stroke II. Evidence from cohort studies, Eur J Clin Nutr, 2004, 58: 718-724.

6 Seidel, C., Deufel, T. and Jahreis, G., Effects of fat-modified dairy products on blood lipids in humans in comparison with other fats, Ann Nutr Metab, 2005, 49: 42-48.

$7 \quad$ Valeille, K., Ferezou, J., Parquet, M., Amsler, G., Gripois, D., QuignardBoulange, A. and Martin, J.-C., The Natural Concentration of the Conjugated Linoleic Acid, cis-9,trans-11, in Milk Fat Has Antiatherogenic Effects in Hyperlipidemic Hamsters, J. Nutr., 2006, 136: 1305-1310.

8 Lindon, J. C., Nicholson, J. K., Holmes, E. and Everett, J. R., Metabonomics: Metabolic Processes Studied by NMR Spectroscopy of Biofluids, Concepts in Magnetic Resonance, 2000, 12: 289-320.

9 Fiehn, O., Metabolomics--the link between genotypes and phenotypes, Plant Mol Biol, 2002, 48: 155-171.

10 Whitfield, P. D., German, A. J. and Noble, P. J., Metabolomics: an emerging postgenomic tool for nutrition, Br J Nutr, 2004, 92: 549-555.

11 Fardet, A., Canlet, C., Gottardi, G., Lyan, B., Llorach, R., Remesy, C., Mazur, A., Paris, A. and Scalbert, A., Whole-Grain and Refined Wheat Flours Show Distinct Metabolic Profiles in Rats as Assessed by a 1H NMR-Based Metabonomic Approach, J. Nutr., 2007, 137: 923-929.

12 Rezzi, S., Ramadan, Z., Fay, L. B. and Kochhar, S., Nutritional metabonomics: applications and perspectives, J Proteome Res, 2007, 6: 513-525.

13 Gibney, M. J., Walsh, M., Brennan, L., Roche, H. M., German, B. and van Ommen, B., Metabolomics in human nutrition: opportunities and challenges, Am. J. Clin. Nutr., 2005, 82: 497-503.

14 Wang, Y., Tang, H., Nicholson, J. K., Hylands, P. J., Sampson, J. and Holmes, E., A metabonomic strategy for the detection of the metabolic effects of chamomile (Matricaria recutita L.) ingestion, J Agric Food Chem, 2005, 53: 191-196.

15 Stella, C., Beckwith-Hall, B., Cloarec, O., Holmes, E., Lindon, J. C., Powell, J., van der Ouderaa, F., Bingham, S., Cross, A. J. and Nicholson, J. K., Susceptibility of human metabolic phenotypes to dietary modulation, J Proteome Res, 2006, 5: 2780-2788. 16 Brindle, J. T., Antti, H., Holmes, E., Tranter, G., Nicholson, J. K., Bethell, H. W., Clarke, S., Schofield, P. M., McKilligin, E., Mosedale, D. E. and Grainger, D. J., Rapid 
and noninvasive diagnosis of the presence and severity of coronary heart disease using 1H-NMR-based metabonomics, Nat Med, 2002, 8: 1439-1444.

17 Ala-Korpela, M., Critical evaluation of $1 \mathrm{H}$ NMR metabonomics of serum as a methodology for disease risk assessment and diagnostics, Clin Chem Lab Med, 2008, 46: $27-42$.

18 Kirschenlohr, H. L., Griffin, J. L., Clarke, S. C., Rhydwen, R., Grace, A. A., Schofield, P. M., Brindle, K. M. and Metcalfe, J. C., Proton NMR analysis of plasma is a weak predictor of coronary artery disease, Nat Med, 2006, 12: 705-710.

19 Valeille, K., Ferezou, J., Amsler, G., Quignard-Boulange, A., Parquet, M., Gripois, D., Dorovska-Taran, V. and Martin, J. C., A cis9,trans11-conjugated linoleic acid rich-oil reduces the outcome of atherogenic process in hyperlipidemic hamster, Am J Physiol Heart Circ Physiol, 2005, 289: H652-H659.

20 Dorfman, S. E., Smith, D. E., Osgood, D. P. and Lichtenstein, A. H., Study of Diet-Induced Changes in Lipoprotein Metabolism in Two Strains of Golden-Syrian Hamsters, J. Nutr., 2003, 133: 4183-4188.

21 Eggen, D. A., Strong, J. P., Newman, W. P., Malcom, G. T. and Restrepo, C., Regression of experimental atherosclerotic lesions in rhesus monkeys consuming a high saturated fat diet, Arteriosclerosis, 1987, 7: 125-134.

22 Kuksis, A., Myher, J. J. and Geher, K., Quantitation of plasma lipids by gas-liquid chromatography on high temperature polarizable capillary columns, J. Lipid Res., 1993, 34: 1029-1038.

23 Meiboom, S. and Gill, D., Rev Sci Instrum, 1958, 29: 688-691.

24 Hurd, R. E., J Magn Reson, 1990, 87: 422-428.

25 Kay, L. E., Keifer, P. and Saarinen, T., Pure absorption gradient enhanced heteronuclear single quantum correlation spectroscopy with improved sensitivity, J Am Oil Chem Soc, 1992, 114: 10663-10665.

26 Gavaghan, C. L., Wilson, I. D. and Nicholson, J. K., Physiological variation in metabolic phenotyping and functional genomic studies: use of orthogonal signal correction and PLS-DA, FEBS Lett, 2002, 530: 191-196.

27 Caraux, G. and Pinloche, S., Permutmatrix: A Graphical Environment to Arrange Gene Expression Profiles in Optimal Linear Order, Bioinformatics, 2005, 21: 1280-1281. 28 Chavance, M., Jackknife and bootstrap, Rev Epidemiol Sante Publique, 1992, 40: 209-218.

29 Lundberg, B., Chemical composition and physical state of lipid deposits in atherosclerosis, Atherosclerosis, 1985, 56: 93-110.

30 Mensink, R. P., Zock, P. L., Kester, A. D. and Katan, M. B., Effects of dietary fatty acids and carbohydrates on the ratio of serum total to HDL cholesterol and on serum lipids and apolipoproteins: a meta-analysis of 60 controlled trials, Am J Clin Nutr, 2003, 77: 1146-1155.

31 Dorfman, S. E., Wang, S., Vega-Lopez, S., Jauhiainen, M. and Lichtenstein, A. H., Dietary Fatty Acids and Cholesterol Differentially Modulate HDL Cholesterol Metabolism in Golden-Syrian Hamsters, J Nutr, 2005, 135: 492-498.

32 Nicholson, J. K., Foxall, P. J., Spraul, M., Farrant, R. D. and Lindon, J. C., 750

MHz 1H and 1H-13C NMR spectroscopy of human blood plasma, Anal Chem, 1995, 67: 793-811. 
33 Mayr, M., Chung, Y.-L., Mayr, U., Yin, X., Ly, L., Troy, H., Fredericks, S., Hu, Y., Griffiths, J. R. and Xu, Q., Proteomic and Metabolomic Analyses of Atherosclerotic Vessels From Apolipoprotein E-Deficient Mice Reveal Alterations in Inflammation, Oxidative Stress, and Energy Metabolism, Arteriosclerosis, Thrombosis and Vascular Biology, 2005, 25: 2135-2142.

34 Patsch, J. R., Miesenbock, G., Hopferwieser, T., Muhlberger, V., Knapp, E., Dunn, J. K., Gotto, A. M., Jr. and Patsch, W., Relation of triglyceride metabolism and coronary artery disease. Studies in the postprandial state, Arterioscler Thromb, 1992, 12: 1336-1345.

35 Bell, J. D., Brown, J. C., Nicholson, J. K. and Sadler, P. J., Assignment of resonances for 'acute-phase' glycoproteins in high resolution proton NMR spectra of human blood plasma, FEBS Lett, 1987, 215: 311-315.

36 Pepys, M. B., CRP or not CRP? That is the question, Arterioscler Thromb Vasc Biol, 2005, 25: 1091-1094.

37 Ahmed, N. and Thornalley, P. J., Chromatographic assay of glycation adducts in human serum albumin glycated in vitro by derivatization with 6-aminoquinolyl-Nhydroxysuccinimidyl-carbamate and intrinsic fluorescence, Biochem J, 2002, 364: 15-24. 38 Oorni, K., Pentikainen, M. O., Annila, A. and Kovanen, P. T., Oxidation of low density lipoprotein particles decreases their ability to bind to human aortic proteoglycans. Dependence on oxidative modification of the lysine residues, J Biol Chem, 1997, 272: 21303-21311.

39 al-Waiz, M., Mikov, M., Mitchell, S. C. and Smith, R. L., The exogenous origin of trimethylamine in the mouse, Metabolism, 1992, 41: 135-136.

40 Rebouche, C. J. and Seim, H., Carnitine metabolism and its regulation in microorganisms and mammals, Annu Rev Nutr, 1998, 18: 39-61.

41 Kahlon, T. S., Chow, F. I., Irving, D. W. and Sayre, R. N., Cholesterol response and fatty streak formation in hamsters fed two levels of saturated fat and various levels of cholesterol, Nutr. Res., 1997, 17: 1693-1707.

42 Ntanios, F. Y., van de Kooij, A. J., de Deckere, E. A., Duchateau, G. S. and Trautwein, E. A., Effects of various amounts of dietary plant sterol esters on plasma and hepatic sterol concentration and aortic foam cell formation of cholesterol-fed hamsters, Atherosclerosis, 2003, 169: 41-50. 
Figure legends

Figure 1. Relative cholesteryl ester (CE) deposition in the whole aorta, with individually marked histograms $(*, \dagger, \dagger)$ displaying statistically different results $(P<0.05$, ANOVA). Results are expressed as the mean $\pm \mathrm{SD}, n=$ six to eight individuals per group. $\mathrm{CR}$, raw milk cheese diet; $\mathrm{CS}$, standard (pasteurized) milk cheese diet; $\mathrm{CO}$, olein fraction cheese diet; Butter oil, extracted pasteurized milk fat; $\mathrm{CV}$, vegetable cheese diet; $\mathrm{C}$, control diet.

Figure 2. Characterization of the plasma metabolic changes induced by dietary challenges. Two-dimensional PLS-DA score plot showing group discrimination according to dietary treatments; percentage of variation of the NMR variables $\left(R^{2} \mathrm{X}\right)$ explained by each component $t$ is indicated. The group assignment for individual hamsters was calculated using six significant components to explain $73 \%$ of metabolite variations. CR, raw milk cheese diet; CS, standard (pasteurized) milk cheese diet; CO, olein fraction cheese diet; Butter oil, extracted pasteurized milk fat; $\mathrm{CV}$, vegetable cheese diet; $\mathrm{Ctrl}$, control diet. $n=$ eight hamsters per group.

Figure 3. Relationship between diet-induced plasma metabolic changes and atherogenesis. Changes among the metabolic score values ( $\mathrm{t}[1])$ of individual hamsters fed various diets are plotted along with the corresponding individual cholesteryl ester deposition scores (u[1]), as determined by PLS analysis (A). The coefficient of determination $\left(R^{2}=0.62\right)$ among $\mathrm{t}[1]$ and $\mathrm{u}[1]$ that were calculated after linearization of the relationship is indicated. Hierarchical clustering of the highly correlated NMR variables to cholesteryl ester deposition in the hamsters fed various diets was performed using the unweighted pair-group method average using the linkage rule and Pearson correlation coefficient as distance (B). The mean metabolite intensity signals among the hamsters are displayed by a heatmap (red boxes: increase, green boxes: decrease, black boxes: no relative change) with rows describing metabolites and columns describing individual hamsters (see Online Supplemental Table 6 for statistical differences among metabolite score values). The index of determination $\left(\mathrm{R}^{2}\right.$ value) among the selected NMR signals and whole aortic CE deposition is presented as a line plot. The combined $\mathrm{R}^{2}$ is calculated from a multiple regression model fitting the values of the metabolites to that of the cholesteryl ester loading in the aorta. The variables of importance in the projection (VIP) allow for metabolite ranking to explain the atherogenic outcome. $n=$ six to eight per group. CR, raw milk cheese diet; CS, standard (pasteurized) milk cheese diet; CO, olein fraction cheese diet; Butter oil, extracted pasteurized milk fat; $\mathrm{CV}$, vegetable cheese diet; $\mathrm{C}$, control diet.

Table 1.

Physiological outcomes of hamsters fed with different diets after 12 weeks of nutritional challenge $^{1,2}$.

\begin{tabular}{|c|c|c|}
\hline $\begin{array}{l}\text { Physiological } \\
\text { and } \\
\text { biochemical } \\
\text { outcomes }\end{array}$ & diets & $\begin{array}{l}R^{2} \text { values } \\
\text { to whole } \\
\text { aorta } \mathrm{CE}\end{array}$ \\
\hline
\end{tabular}

Control CV diet CS diet CR diet CO diet Butter




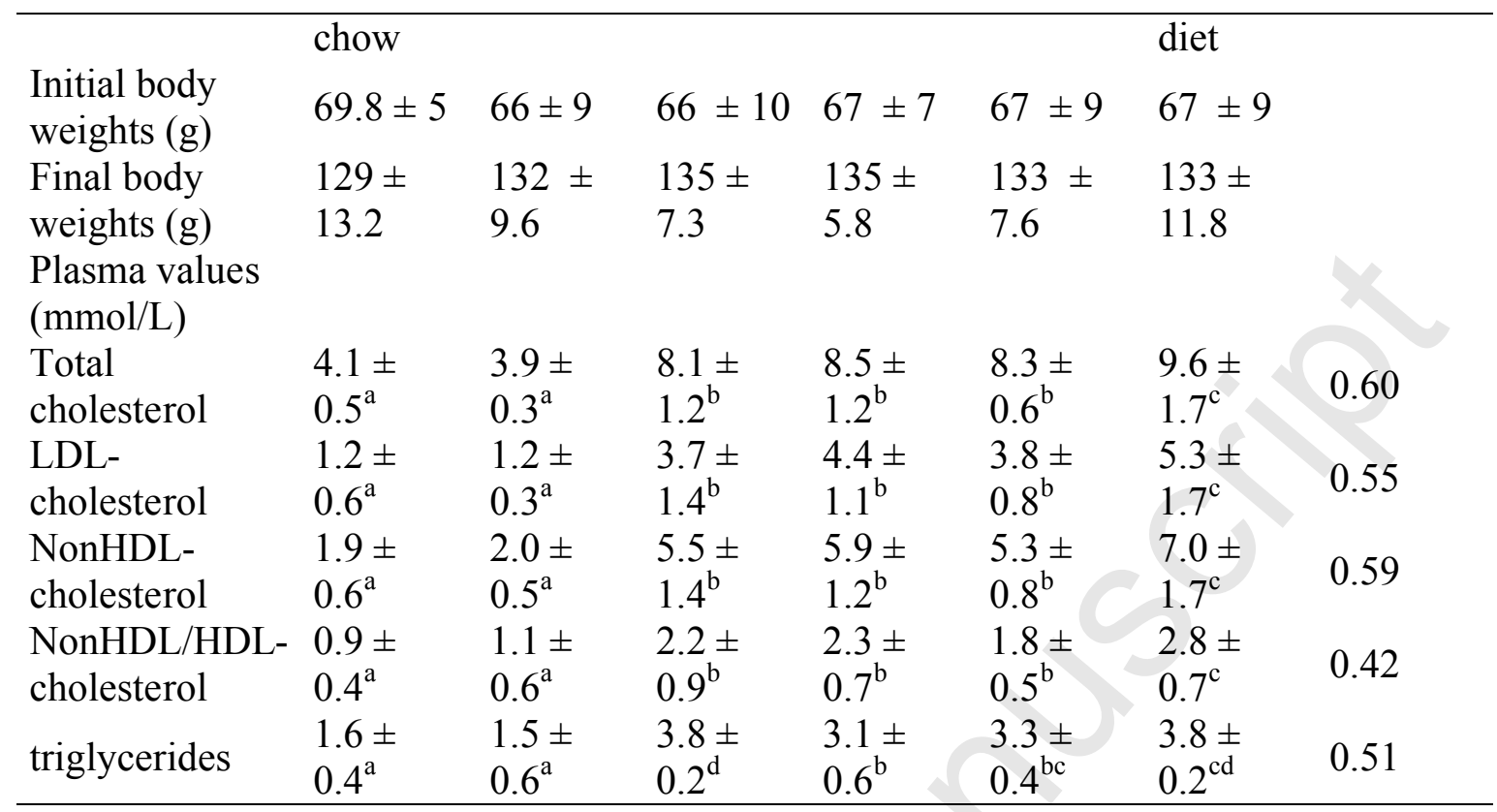

${ }^{1}$ Results are expressed as mean $\pm \mathrm{SD} ; n=8$ hamsters per group ; means in a raw not sharing a common superscript letter differ (one way ANOVA followed by a post-hoc Fisher test, $P<0.05)$.

${ }^{2} \mathrm{CV}$, vegetable cheese-based diet; CR, raw milk cheese-based diet; CS, standard pasteurized cheese-based diet; $\mathrm{CO}$, milk fat olein fraction-based diet. 
Figure 1

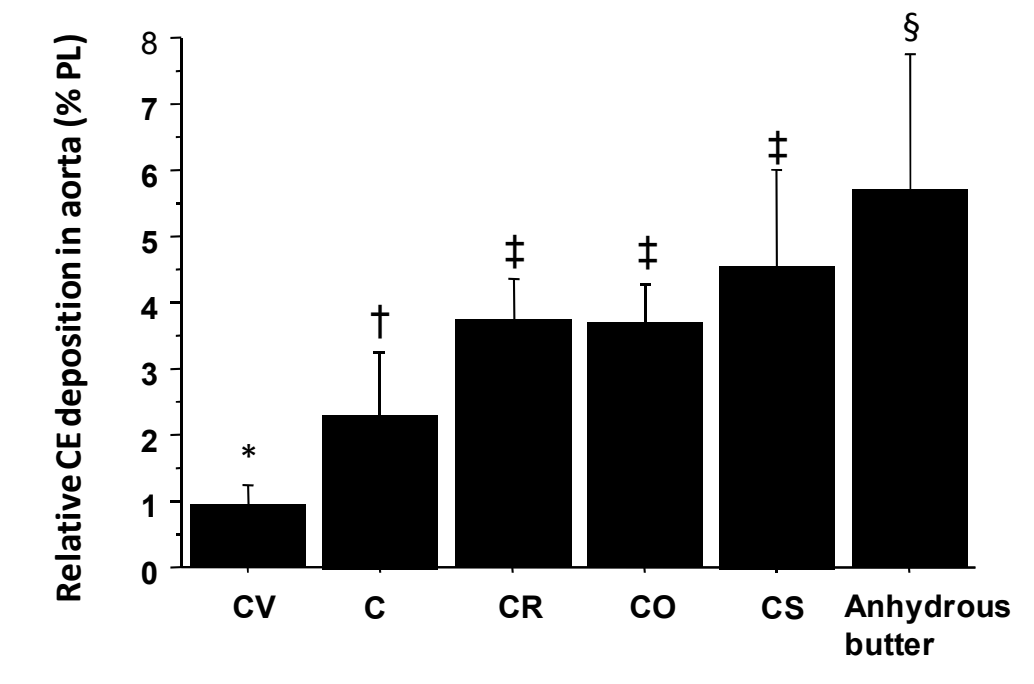


Figure 2

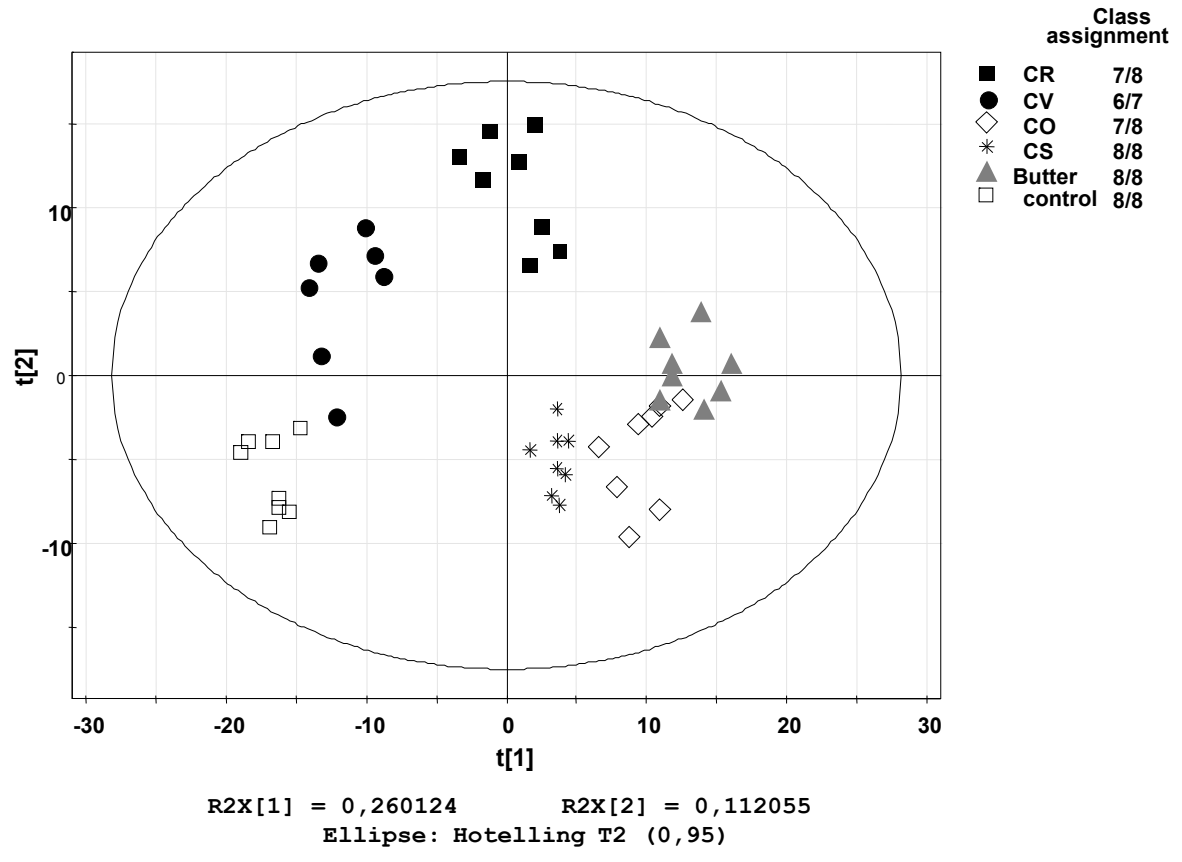


Figure 3

Figure 3

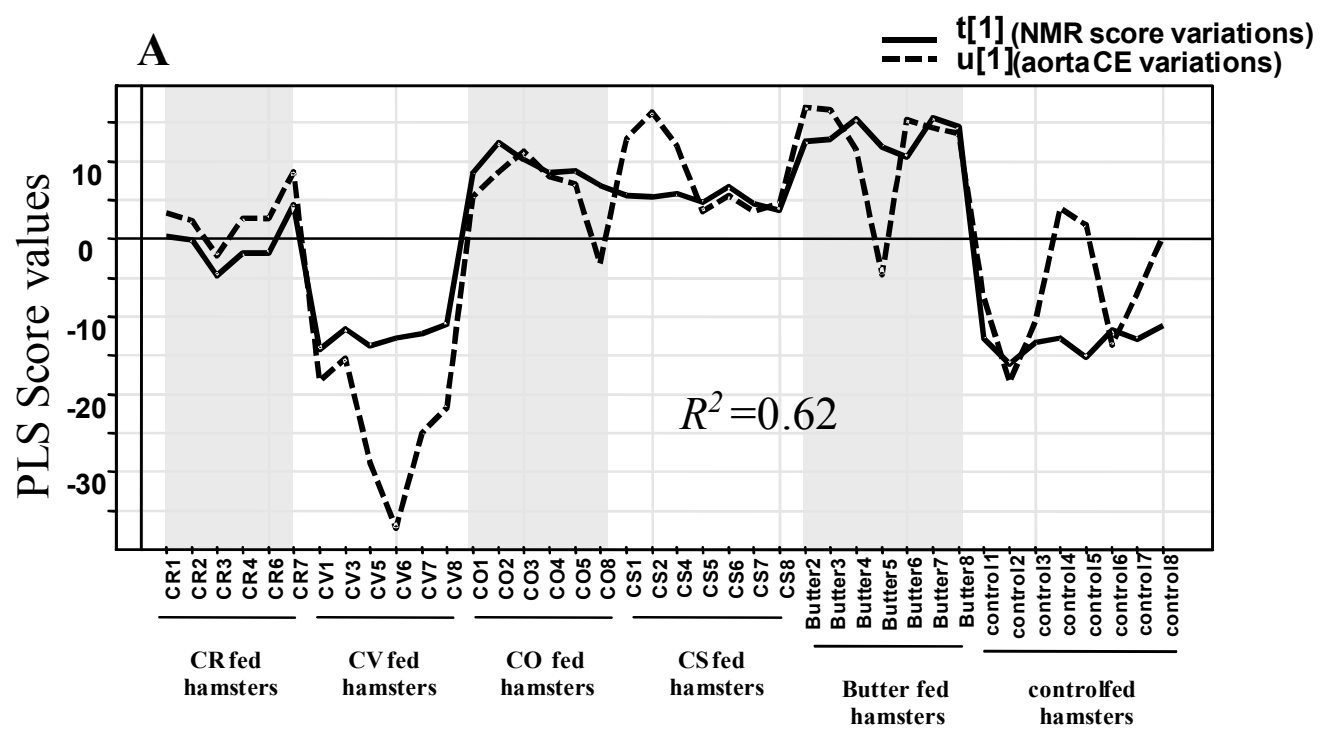

B

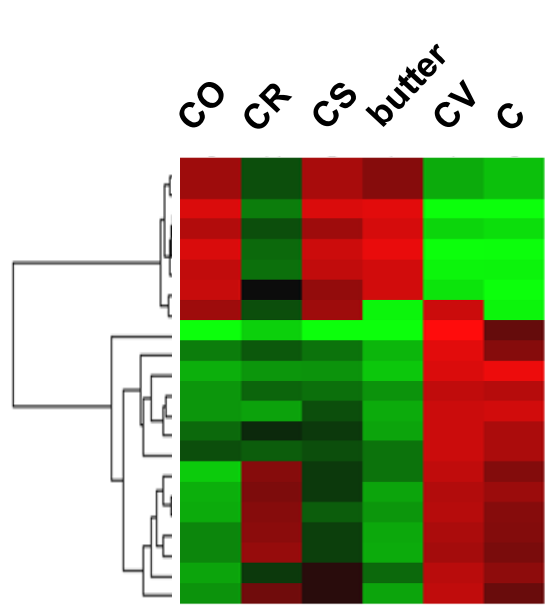<smiles>C#CC=C</smiles>

Individual metabolites $R \mathbf{2}$ values to whole aorta CE

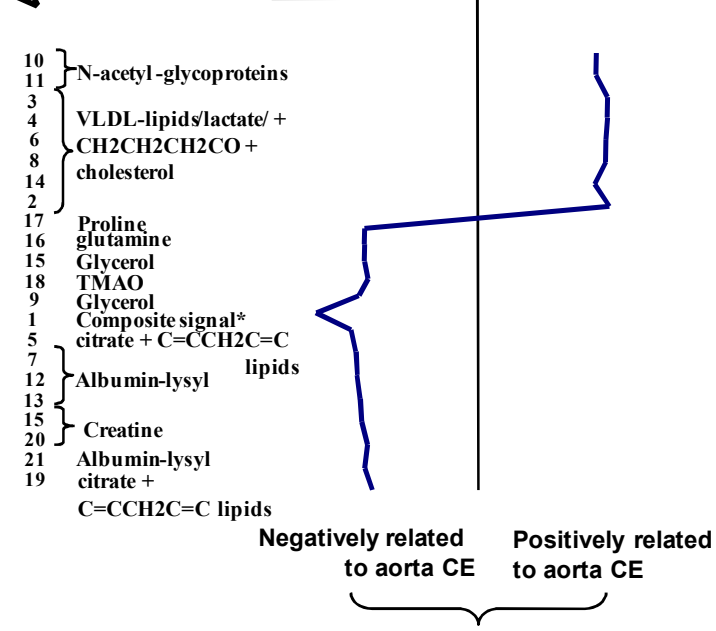

* Alanine/glu tamine/glucose/unassigned

Combined $R 2=0.89$ 